\title{
Non-syndromic Sensorineural Prelingual Deafness: The Importance of Genetic Counseling in Demystifying Parents' Beliefs About the Cause of Their Children's Deafness
}

\author{
Fidjy Rodrigues • Milena Paneque • Cláudia Reis • \\ Margarida Venâncio • Jorge Sequeiros • Jorge Saraiva
}

Received: 25 October 2011 / Accepted: 20 December 2012

(C) National Society of Genetic Counselors, Inc. 2013

\begin{abstract}
Recent advances in molecular genetics have allowed the determination of the genetic cause of some childhood non-syndromic deafness. In Portugal only a small proportion of families are referred to a clinical genetics service in order to clarify the etiology of the deafness and to provide genetic counseling. Consequently, there are no published studies of the prior beliefs of parents about the causes of hereditary deafness of their children and their genetic knowledge after receipt of genetic counseling. In order to evaluate the impact of genetic counseling, 44 parents of 24 children with the diagnosis of non-syndromic sensorineural prelingual deafness due to mutations in the GJB2 (connexin 26), completed surveys before and after genetic counseling. Before counseling $13.6 \%$ of the parents knew the cause of deafness; at a post-counseling setting this percentage was significantly higher, with $84.1 \%$ of the parents accurately identifying the etiology. No significant differences were found between the
\end{abstract}

F. Rodrigues $\cdot$ M. Paneque $\cdot J$. Sequeiros

ICBAS, Universidade do Porto, Porto, Portugal

F. Rodrigues $(\bowtie) \cdot C$. Reis $\cdot$ M. Venâncio $\cdot J$. Saraiva

Serviço de Genética Médica, Hospital Pediátrico

Carmona da Mota, Centro Hospitalar Universitário

de Coimbra, Av. Afonso Romão, Santo António Olivais,

3000-602 Coimbra, Portugal

e-mail: fidjy20@hotmail.com

M. Paneque $\cdot$ J. Sequeiros

IBMC - Instituto de Biologia Molecular e Celular,

Universidade do Porto, Porto, Portugal answers of mothers and fathers either before or after genetic counseling. Parents' level of education was a significant factor in pre-test knowledge. After genetic counseling $95.5 \%$ of the parents stated that the consultation had met their expectations, $70.5 \%$ remembered correctly the inheritance pattern, and $93.2 \%$ correctly recalled the chance of risk of deafness. These results underline the importance of genetic counseling in demystifying parents' beliefs about the etiology of their children's deafness.

Keywords Genetic counseling · Beliefs · Genetic knowledge $\cdot$ Deafness $\cdot$ Portugal

\section{Introduction}

The incidence at birth of childhood deafness is 1:500 newborns. About $50 \%$ of these cases have a genetic cause; rising to $70 \%$ in non-syndromic cases (isolated deafness) of these - 75-80\% have an autosomal recessive inheritance (Smith et al. 2010). Genetic conditions have long been proposed to have social and psychological consequences (worry about the health of children, feelings of stigmatization, and guilt) which could affect the family's ability to adjust to the condition (James et al. 2006). After the birth of a deaf child, parents are usually unaware of the etiology of the deafness and the majority does not expect a genetic etiology when there was no explicit family history of this condition (Steinberg et al. 2007). Consequently, they search for explanations for this unexpected event, but it often takes 
them a long time to find accurate information (Brunger et al. 2000).

Following a correct clinical assessment of the type of deafness, referral to a genetics department for genetic counseling is extremely important. Genetic counseling appropriately focuses on the needs of individual families, with counselors providing information in a supportive environment and according to the counselee's psychosocial needs, cultural differences and prior knowledge, all of which may influence communication and decision-making processes (Kaimal et al. 2007). In this setting parents can be informed of the genetic etiology, be able to better understand the meaning of genetics concepts, inheritance patterns and the chance of recurrence, and also be assisted in their coping process. Moreover, identification of the causative gene and mutations is required for provide a prenatal or pre-implantation genetic diagnosis to the parents (Dagan et al. 2002). Several studies have evaluated parental attitudes toward genetic testing for pediatric deafness, including prenatal diagnosis. The data demonstrated the importance of an accurate genetic counseling and indicated the great interest of the parents in prenatal genetic testing (Abe et al. 2010; Brunger et al. 2000; Kaimal et al. 2007; Li et al. 2007; Palmer et al. 2009; Steinberg et al. 2007).

In Portugal only a small proportion of families are actually referred to a clinical genetics center in order to clarify the etiology of the deafness and to receive genetic counseling. Nonetheless, it is important to take into account parents' beliefs about the cause of their children's deafness and assess their genetic knowledge, in order to improve the quality of the communication process and provide them with the opportunity to make informed decisions.

\section{Purpose of the Present Study}

The aims of this study were to: (1) assess genetic knowledge and beliefs regarding the causes of nonsyndromic sensorineural prelingual deafness of Portuguese parents with a deaf child, both before and after genetic counseling; (2) examine the relationship between the information provided and parents' demographic characteristics; (3) evaluate their satisfaction with the genetic counseling session; and (4) evaluate any feelings of guilt.

\section{Methods}

Participants and Procedures

The Ethics Committee of our Hospital approved all of the procedures, and we obtained written informed consent from each individual. The study was performed during January 2011.

Participants were recruited over a period of 1 month after being referred by an otorhinolaryngologist to our medical genetics department. These families were referred for the first session of genetic counseling at the medical genetics department (Hospital Pediátrico Carmona da Mota, Centro Hospitalar e Universitário de Coimbra) by an otorhinolaryngologist who ordered molecular testing as part of the diagnostic evaluation. A single clinical geneticist, according to the standard protocol of our department, offered genetic counseling. The genetic counseling sessions were standardized as much as possible. During the session the medical doctor explains the reason for the consultation, the basis of the deafness, and explains the inheritance pattern and the chance of recurrence of the deafness for their future children, grandchildren, and the children of their extended family members. Reproductive options also are presented. Based in informed choice, the clinical geneticist asked to the parents it they want to pursue genetic testing.

Forty-four parents (24 mothers and 20 fathers) participated in this study. Their characteristics are presented in Table 1 and summarized in the "Results" section.

\section{Instrumentation}

Based on a review of literature, we developed a semistructured and self-completion questionnaire primarily to assess parents' knowledge and beliefs about the nonsyndromic sensorineural prelingual deafness of their children. The questionnaire was administered before the parents participated in genetic counseling and immediately after the session.

Table 1 Social and demographic characteristics of participating parents $(N=44)$

\begin{tabular}{lcc}
\hline & $n$ & $\%$ \\
\hline Gender & 20 & \\
Male & 24 & 45.5 \\
Female & & 54.5 \\
Educational level & 31 & \\
Primary education & 13 & 70.5 \\
Secondary/higher education & & 29.5 \\
Marital status & 13 & \\
Single/divorced & 31 & 29.5 \\
Married/living with a partner & & 70.5 \\
Geographical area & 8 & 18.2 \\
North & 21 & 47.7 \\
Centre & 15 & 34.1 \\
South & & \\
\hline
\end{tabular}




\section{Pre-genetic Counseling Questionnaire}

The pre-counseling questionnaire contained questions eliciting demographic information about the parent participants (gender, age, geographical area, educational level, marital status), and demographic information about their children with deafness (gender, age and date of cochlear implant procedure). One question asked parents about the reason for the consultation: Do you know the reason for the consultation? (Yes or No?). Parents who responded "Yes" were then invited to write down the reason. Their responses were grouped into one of two categories: To learn the cause of the deafness, or To know whether the deafness had a genetic cause. The second question assessed parents' previous information about the clinical status of their children: What do you know about the deafness of your children? Content analysis of their responses yielded four categories: No relevant information, Genetic caused deafness, Congenital deafness, and Profound bilateral sensorineural deafness.

In order to evaluate knowledge of etiology of the deafness, parents were asked to check one option from a checklist of 15 options. They were asked to endorse one option at two points in time - pre and post counseling. The options are: Fate, Use chemicals or pollution during pregnancy, Relevant family history in the father's family, Relevant family history in the mother's family, Medication during mother's pregnancy, Alcohol or drugs during mother's pregnancy, Accident during the pregnancy, A genetic variant in a gene or chromosome of parents, As a punishment, Child had an accident or injury in the neonatal period or childhood, Medical error, Either mother or father smoked during the pregnancy, God's will, History of deafness in the family, I do not know.

In order to evaluate feelings of guilt based on their beliefs about the causes of their children's deafness, participants responded to the following item: Do you think you could do something to prevent the deafness of your child? (Scale: $1=$ Completely agree, 2 = Agree, $3=$ Disagree, $4=$ Completely disagree). They were asked to respond to this item at two points in time - pre and post counseling.

In order to evaluate the effects of their knowledge and beliefs on their perceived reproductive options, participants responded to the following item: If I had more information my attitude towards having more children would have changed? (Scale: 1 = Completely agree, $2=$ Agree, $3=$ Disagree, $4=$ Completely disagree). They were asked to respond to this item at two points in time-pre and post counseling.

\section{Post-genetic Counseling Questionnaire}

In addition to some of the items from the pre-genetic counseling questionnaire, parents answered questions that assessed their opinion about the genetic counseling session: "Did the genetic counseling meet your expectations?" and
"Was the genetic counseling important to understand the cause of the children's deafness?". We also evaluated recall of other information: knowledge of recurrence risk was tested by a multiple-choice question with the following options: $50 \%, 25 \%$, and I do not know. The same format was used to assess knowledge about the inheritance pattern by presenting the following options: Autosomal Dominant, Autosomal Recessive, and I do not know. We only considered the inheritance patterns that are most frequent and were applicable to our patient population.

\section{Statistical Analysis}

Data were analyzed with the Statistical Programs for the Social Sciences (SPSS), version 19.0 for Windows. Descriptive statistics were calculated for the demographic variables as well as the causes of non-syndromic pre-lingual deafness, pre and post-counseling. Chi-square tests were performed to determine significant relationships between pre and post-counseling answers, and participants' gender and their educational level (primary education or secondary/ higher education). A dependent t-test was conducted to determine if there were any statistically significant changes in pre and post-counseling beliefs. A value of $p<.05$ was considered statistically significant.

\section{Results}

\section{Sample Demographic and Social Characteristics}

Table 1 contains a summary of the sample's demographic and social characteristics. We evaluated 44 parents of 24 children with a profound bilateral sensorineural deafness. All these families are genetically distinct. The children were homozygous or compound heterozygotes for mutations in the GJB2 (connexin 26). The mean age of these children was 11.8 years $(\mathrm{SD}=3.6$ years). All underwent cochlear implant due to nonsyndromic sensorineural prelingual deafness (mean age at cochlear implant $=32.7$ months, $\mathrm{SD}=7.5$ ). The mean age of the sample of 44 parents was 39.4 years $(\mathrm{SD}=6.9$ years). Most $(70.5 \%, n=31)$ had a primary education, while the remaining parents $(n=13)$ had a secondary/higher education. A majority $(70.5 \%, n=31)$ were either married or living with a partner. None of the parent participants presented with deafness.

Pre and Post Counseling Evaluation

\section{Reason for the Consultation}

Before genetic counseling, 68.1\% (30/44) of the parents knew the reason why the consultation was recommended. Of those 30 parents, 17 (38.6\%) stated it was in order to 
know if the deafness had a genetic cause. Evaluation of the parents' previous information about non-syndromic sensorineural prelingual deafness revealed that a small percentage, $15.9 \%(7 / 44)$, was aware that a genetic factor comprised the etiology of the deafness, and $20.5 \%(9 / 44)$ of the participants knew that the deafness had been classified as a profound bilateral sensorineural deafness. Table 2 contains a summary of participants' answers to the following questions: "Do you know the reason for the consultation? Those who answered "Yes" were then asked to: What is the reason? What do you know about the deafness of your children?

\section{Before Genetic Counseling: Knowledge of the Etiology of the Deafness}

Before genetic counseling, the investigators evaluated what the parents believed were the causes for their children's deafness. Table 3 lists 15 possible causes that parents' could choose at pre and post-counseling. Before receiving genetic counseling the statement: "I do not know" was most prevalent $(13 / 44)$. In the sample, 8 of 44 parents believed the deafness of their children was due to the "History of deafness in the family." Seven parents thought that the cause of the deafness could be explained by "An accident or injury in the neonatal period or childhood." At that time, $13.6 \%$ (6/ 44) indicated a genetic cause: "A genetic variant in a gene or chromosome of parents."

\section{After Genetic Counseling: Knowledge of Etiology of the Deafness}

After information was transmitted in the genetic counseling session, 37 of 44 respondents ( $84.1 \%$ ) accurately reported the cause of the deafness, affirming it was due to "A genetic variant in a gene or chromosome of parents." This was a significant change after the genetic counseling session,

Table 2 Parent's responses to questions prior to genetic counseling $(N=44)$

\begin{tabular}{lc}
\hline Questions and arranged answers & $\%$ \\
\hline Do you know the reason for the consultation? & \\
Yes & $68.1 \%(30 / 44)$ \\
No & $31.9 \%(14 / 44)$ \\
Those who answered "Yes" to the above question: What is the reason? & $29.5 \%(13 / 44)$ \\
To learn the cause of the deafness & $38.6 \%(17 / 44)$ \\
To know if the deafness had a genetic cause & $20.5 \%(9 / 44)$ \\
What do you know about the deafness of your children? \\
A congenital deafness & $43.2 \%(19 / 44)$ \\
No relevant information & $15.9 \%(7 / 44)$ \\
Genetic caused deafness & $20.5 \%(9 / 44)$ \\
Profound bilateral sensorineural deafness &
\end{tabular}

$\chi^{2}(1)=40.93, p<.001$. A small percent $(9.1 \%)$ continued to believe that the deafness was caused by the "History of deafness in the family," and 3 of 44 participants (6.8 \%) still did not know the etiology.

\section{Relationships Between the Information Provided and Participant Demographic Variables}

When the investigators evaluated educational differences in the pre-counseling responses of parents they found that a significantly greater percentage of individuals with secondary/higher education (38.5\%) accurately identified the causes of the deafness when compared with individuals with primary education (3.2\%) at pre-counseling, $\chi^{2}(1)=7.69, p=.006$. At post-counseling, $92.3 \%$ of participants with secondary/higher education and $80.6 \%$ of those with primary education provided the correct answer; this difference in percentages due to education level was not significant, $\chi^{2}(1)=0.26, p=.610$. There were no significant differences between fathers and mothers in the percentage that correctly answered at either pre-counseling, $\chi^{2}(1)=0.05, p=.823$ or post-counseling, $\chi^{2}(1)=0.04, p=.841$.

\section{Evaluation of the Genetic Counseling: Satisfaction and Recall of the Information}

After the genetic counseling session, $95.5 \%$ of parents confirmed that the consultation met their expectations and all parents mentioned the relevance of genetic counseling in helping them understand the cause of their children's deafness. All parents agreed to undergo genetic testing in order to confirm the carrier status. About $70.5 \%$ of the parents reported that they understood and remembered correctly the information transmitted in the consultation about the mode of inheritance. They indicated that the deafness has an autosomal recessive inheritance. Nevertheless, $22.7 \%$ (10/ 44) said they did not remember this information. Regarding the chance of recurrence, $93.2 \%$ of the respondents were able to remember correctly the information transmitted and only $2.3 \%$ did not remember this information.

\section{The Feeling of Guilt}

Before and after genetic counseling, parents were asked to indicate their agreement with the question "Do you think you could do something to prevent the deafness of your child?" in order to evaluate possible feelings of guilt. Their mean agreement with the idea that they might have done something different to prevent their child's disease was $3.23(\mathrm{SD}=0.95)$ at pre-counseling, and $2.93(\mathrm{SD}=1.03)$ at post-counseling. The difference between these means was not significant, $\mathrm{t}(42)=1.463, p=.151$. After genetic counseling, 12 of 44 respondents changed their minds from 
Table 3 Probable causes of deafness identified by parents at pre and post-counseling $(N=44)$

Participants were instructed to check only one response option

\begin{tabular}{|c|c|c|c|c|}
\hline & \multicolumn{2}{|c|}{ Pre-counselling } & \multicolumn{2}{|c|}{ Post-counselling } \\
\hline & $n$ & $\%$ & $n$ & $\%$ \\
\hline Fate & 2 & 4.5 & 0 & 0 \\
\hline Use chemicals or pollution during pregnancy & 0 & 0 & 0 & 0 \\
\hline Relevant family history in the father's family & 0 & 0 & 0 & 0 \\
\hline Relevant family history in the mother's family & 2 & 4.5 & 0 & 0 \\
\hline Medication during mother's pregnancy & 5 & 11.4 & 0 & 0 \\
\hline Alcohol or drugs during mother's pregnancy & 0 & 0 & 0 & 0 \\
\hline Accident during the pregnancy & 0 & 0 & 0 & 0 \\
\hline A genetic variant in a gene or chromosome of parents & 6 & 13.6 & 37 & 84.1 \\
\hline As a punishment & 0 & 0 & 0 & 0 \\
\hline Child had an accident or injury in the neonatal period or childhood & 7 & 15.9 & 0 & 0 \\
\hline Medical error & 0 & 0 & 0 & 0 \\
\hline Either mother or father smoked during the pregnancy & 0 & 0 & 0 & 0 \\
\hline God's will & 1 & 2.3 & 0 & 0 \\
\hline History of deafness in the family & 8 & 18.2 & 4 & 9.1 \\
\hline I do not know & 13 & 29.5 & 3 & 6.8 \\
\hline
\end{tabular}

"Completely Disagree"/ "Disagree" to "Agree"/ "Completely Agree" regarding the idea that they might have done something to prevent the child's disease, while 23 of 44 parents did not change their minds, continuing to "Completely Disagree" or "Disagree." Only three parents changed their opinion from "Agree"/ "Completely Agree" to "Completely Disagree/ Disagree."

\section{Discussion}

Previous Knowledge About the Deafness of Their Children

In Portugal this was the first study that evaluated parents' beliefs about the deafness of their children and parents' knowledge of genetic information prior to and after genetic counseling. In our investigation only a small percentage of the parents endorsed the genetic factor as the cause of their children's deafness before genetic counseling, and one-fifth of the participants knew that the deafness had been classified as profound bilateral sensorineural deafness. These parents claimed to have received this information from an otorhinolaryngologist prior to participating in our project. In their study Brunger et al. (2000) observed that parents had a very poor understanding of genetic factors. Abe et al. (2010) described in a previous study that before visiting a genetic department only $36 \%$ of the respondents were aware of a genetic factor as the cause of deafness because they obtained the information from various sources namely another hospital, a friend, a magazine and the internet. Given the poor knowledge of parents before genetic counseling, it is important that genetic counselors spend time assessing their knowledge and beliefs in order to anticipate how each parent will incorporate information into their understanding of their child's deafness and to improve their genetics knowledge. Kaimal et al. (2007) confirmed the need for clinical geneticists and genetic counselors to be aware of and sensitized to the questions and reasons that bring parents to a genetic consultation. Middleton et al. (1998) confirmed that any genetic counseling is only effective and appropriate when clinicians and counselors take into consideration the prior beliefs of counselees. Successful counseling is achieved when families receive accurate information and understand the relevant information. In this sense, we evaluated whether the genetics knowledge of the participants improved after the impact of the genetic counseling.

\section{Genetic Counseling: Evaluation of Genetic Knowledge}

In our study the majority of the participants confirmed that genetic counseling was helpful to understand the etiology of their children's deafness and it met their expectations. Similar data were found in previous studies demonstrating that it is important for parents to know why their children are deaf (Brunger et al. 2000; Dagan et al. 2002; Li et al. 2007; Palmer et al. 2009; Withrow et al. 2008). After counseling, the majority of our participants understood the etiology of the deafness, with the difference between pre and post counseling being significant. The majority answered "A genetic variant in a gene or chromosome of parents" is the reason of the deafness of their children. Baldwin et al. (2011) affirmed that genetics knowledge is enhanced by genetic counseling. Weil (1991) demonstrated 
similar results and affirmed that after genetic counseling the statement "It happened because of something in the genes or chromosomes" received the highest average rating when he questioned parents about the causes of their children's genetic disorders.

Nevertheless, in our study after counseling a small percentage of individuals still could not identify the etiology. Abe et al. (2010) reported similar data in their study, where $14 \%$ of the respondents who received an explanation did not fully understand it. This difficulty may be related to the low educational level of many of our participants. Steinberg et al. (2007) found that parents with less formal education had more misconceptions about the process of genetic transmission and they concluded that it is necessary to transmit information according to the needs of parents based on their prior knowledge and education level. Several studies confirmed that age and education level were consistent demographic predictors of genetics knowledge (Baldwin et al. 2011; Cabrera et al. 2010; Kaimal et al. 2007; Kelly et al. 2004; Seidenfeld and Antley 1981). We obtained similar results in our study, as a significantly greater percentage of parents with secondary/higher education correctly identified correctly answers about the etiology of deafness than did parents with primary education at pre-counseling. Some parents without high school education had more difficulties understanding the test results and its related terms. At postcounseling, $92.3 \%$ of the individuals with secondary/higher education and $80.6 \%$ of those with primary education identified the correct answer. These findings confirm that it is necessary to transmit information according to the needs of parents based on their knowledge and education level. Genetic counselors may need to adjust their language in order to be more fully understood by their patients.

\section{Recall of Information: The Chance of Recurrence and the Inheritance Pattern}

The results of the current study demonstrate that appropriate genetic counseling can help parents to understand the chance of recurrence and the mode of inheritance. This information had not been available for the majority of parents until the day of the genetic consultation. The participants were satisfied with receiving information regarding recurrence risk for their future children, grandchildren and the children of extended family members who might have deafness. At the end of the appointment, the majority could accurately identify the recurrence risk and the inheritance pattern. These data demonstrate not only the effectiveness of the consultation but also the importance of the counseling being done by someone with clinical genetics training. Somer et al. (1988) obtained similar results in an earlier study where $80 \%$ of individuals or couples counseled between 1972 and 1981 showed adequate knowledge about the mode of inheritance and $74 \%$ about the chance of recurrence. Other reports indicate that parents can understand concepts of inheritance, genetic origin and the chance of recurrence after genetic counseling (Abe et al. 2010; Michie et al. 1997; Palmer et al. 2009). In contrast, Brunger et al. (2000) found that parents had a very poor understanding of the inheritance of deafness, and little understanding of genetic mechanisms and recurrence risk, suggesting they may not have received genetic counseling or that the genetic counseling they received was inadequate.

\section{Feeling of Guilt}

In addition to parents' beliefs about the etiology of the hearing loss, we evaluated their feelings of guilt, since this emotion has been frequently reported in the literature (Brunger et al. 2000; Kessler et al. 1984; Steinberg et al. 2007; Weil 2000; Withrow et al. 2008). In our study the majority of parents did not change their minds about the idea that they might have done something to prevent their child's deafness, disagreeing before and after genetic counseling. Thus, this sample of parents did not appear to feel guilt about the clinical condition of their children. These data are in accordance with earlier investigations (Lenhard et al. 2005) showing that parents of children with a genetic condition identified less guilt than parents of children with mental retardation or multiple congenital anomalies of unknown etiology. Nevertheless, in all of these cases it is important for genetic counselors to provide an opportunity for parents to express their feelings (whether guilt or some other emotion) and to assure them that these feelings are common and normal.

\section{Study Limitations}

Certain limitations of the study should be noted, namely the small sample which may affect the generalizability of the results. The sample was limited because the investigators were under time restrictions for conducting the study. The inclusion of deaf parents may have yielded additional interesting findings. Unfortunately we did not have not sufficient resources, namely, interpreters, to provide genetic counseling to deaf people. Another limitation concerns the pre-post nature of the study. Based on this design, it cannot be determined whether parents' understanding of information such as etiology and recurrence risk would be retained over time.

\section{Conclusions and Research Recommendations}

Many studies have evaluated the beliefs and attitudes of parents about genetic testing of children with deafness. 
Nevertheless no published study in Portugal has specifically assessed prior beliefs of parents regarding the deafness of their children and their knowledge of genetics after genetic counseling offered by professionals with specialized training. In the present study, the majority of parents did not know the etiology of their children's deafness until after receiving genetic counseling. The present data demonstrated increased knowledge at post-counseling and satisfaction of every participant with the appointment. Thus, genetic counseling seems to be extremely important for parents to help them understand the cause of their children's deafness, the chance of recurrence for their family members, and the mode of inheritance. The results establish the importance of accurate and specific genetic counseling to enhance parents' knowledge of the etiology of the deafness. It is important that genetic counselors spend time discussing the knowledge and beliefs of families to better meet their needs, to anticipate how each individual will incorporate information into their understanding of a child's deafness, and to improve their genetics knowledge. These facts should be transmitted according to the individual needs of parents based on their knowledge and education level. Successful counseling is achieved when families understand the information and the genetic counselors enable the expression of the parents' emotions and doubts.

Nonetheless we need to be careful with the interpretation of the present data due to the sample size. A future study in a national context may help to validate the findings and also generate additional data about the long term impact of genetic counseling on feelings of guilt, reproductive options, and retention and comprehension of the information transmitted in the genetic counseling session. Additionally, it would be important in Portugal to develop genetic counseling guidelines for the etiologic diagnosis of congenital deafness, in order to offer earlier genetic counseling to affected families.

Acknowledgments This study was conducted as part of the Master's project of the first author. This work was produced within the framework of a research seminar of the Professional Masters Course in Genetic Counseling of ICBAS, Universidade do Porto.

The authors wish to express a special acknowledgement to the families who participated in this research.

\section{References}

Abe, S., Noguchi, Y., \& Kitamura, K. (2010). What do patients with hereditary deafness think of genetic studies? Auris, Nasus, Larynx, 37, 422-426.

Baldwin, E.E., Boudreault, P., Fox, M., Sinsheimer, J.S., Palmer, C.G.S. (2011). Effect of pre-test genetic counseling for deaf adults on knowledge of genetic testing. Journal of Genetic Counseling, 5, [Epub ahead of print].

Brunger, J. W., Murray, G. S., O’Riordan, M., Matthews, A. L., Smith, R. J., \& Robin, N. H. (2000). Parental attitudes toward genetic testing for paediatric deafness. American Journal of Human Genetics, 67, 1621-1625.

Cabrera, E., Blanco, I., Yague, C., \& Zabalegui, A. (2010). The impact of genetic counseling on knowledge and emotional responses in Spanish population with family history of breast cancer. Patient Education and Counseling, 78, 382-388.

Dagan, O., Hochner, H., Levi, H., Raas-Rothschild, M., \& Sagi, M. (2002). Genetic testing for deafness: different motivations for the same outcome. American Journal of Medical Genetics, 113, 137143.

James, C., Hadley, D., Holtzman, A., \& Winkelstein, A. (2006). How does the mode of inheritance of a genetic condition influence families? A study of guilt, blame, stigma, and understanding of inheritance and reproductive risks in families with X-linked and autosomal recessive diseases. Genetics in Medicine, 8, 234-242.

Kaimal, G., Steinberg, A. G., Ennis, S., Harasink, S. M., Ewing, R., \& Li, Y. (2007). Parental narratives about genetic testing for deafness: a one year follow up study. Journal of Genetic Counseling, 16, 775-787.

Kelly, K., Leventhal, H., Marvin, M., Toppmeyer, D., Baran, J., \& Schwalb, M. (2004). Cancer genetics knowledge and beliefs and receipt of results in Ashkenazi Jewish individuals receiving counseling for BRCA1/2 mutations. Cancer Control, 11(4), 236-244.

Kessler, S., Kessler, H., \& Ward, P. (1984). Psychological aspects of genetic counseling. III. Management of guilt and shame. American Journal of Medical Genetics, 17, 673-697.

Lenhard, W., Breitenbach, E., Ebert, H., Schindelhauer-Deutscher, H. J., \& Henn, W. (2005). Psychological benefit of diagnostic certainty for mothers of children with disabilities: lessons from Down syndrome. American Journal of Medical Genetics, 133(A), 170-175.

Li, Y., Steinberg, A. G., Bain, L., Yaeger, D., Bieler, A., Erwing, R., et al. (2007). Assessing parental attitudes for childhood deafness: before and after genetic consultation. American Journal of Medical Genetics, 143(A), 1546-1553.

Michie, S., McDonald, V., \& Marteau, T. M. (1997). Genetic counseling: information given, recall and satisfaction. Patient Education and Counseling, 32, 101-106.

Middleton, A., Hewison, R., \& Mueller, F. (1998). Attitudes of deaf adults toward genetic testing for hereditary deafness. American Journal of Human Genetics, 63, 1175-1180.

Palmer, C. G. S., Martinez, A., Fox, M., Zhou, J., Shapiro, N., Sininger, Y., et al. (2009). A prospective, longitudinal study of the impact of $G J B 2 / G J B 6$ genetic testing on the beliefs and attitudes of parents of deaf and hard-of-hearing infants. American Journal of Medical Genetics, 149(A), 1169-1182.

Seidenfeld, M. J., \& Antley, R. M. (1981). Genetic counselling: a comparison of counselee's genetic knowledge before and after. American Journal of Medical Genetics, Part III, 10, 107-112.

Smith, R., Hildebrand, M., Van Camp, G. (2010). Deafness and hereditary deafness overview. http://www.ncbi.nlm.nih.gov/pubmed/ 20301607. Accessed 7 January 2011.

Somer, M., Mustonen, H., \& Norio, R. (1988). Evaluation of genetic counselling: recall of information, post-counselling reproduction, and attitude of the counselees. Clinical Genetics, 34, 352-365.

Steinberg, A. G., Kaimal, G., Bain, L., Krantz, I., \& Li, Y. (2007). Parental narratives on genetic testing for children with deafness: a qualitative inquiry. American Journal of Medical Genetics, 143(A), $1533-1545$.

Weil, J. (1991). Mother's postcounseling beliefs about the causes of their children's genetic disorders. American Journal of Human Genetics, 48, 145-153.

Weil, J. (2000). Psychosocial genetic counseling. New York, NY: Oxford University Press.

Withrow, K. A., Burton, S., Arnos, K. S., Kalfoglou, A., \& Pandya, A. (2008). Consumer motivations for pursuing genetic testing and their preferences for the provision of genetic services for deafness. Journal of Genetic Counseling, 17, 252-260. 